\title{
Valsartan Attenuates Atherosclerosis via Upregulating the Th2 Immune Response in Prolonged Angiotensin II-Treated ApoE $^{-/-}$Mice
}

\author{
Kai Meng ${ }^{1,2^{*}}$ Qiutang Zeng, ${ }^{2 *}$ Qinghua Lu, ${ }^{1^{*}}$ Yingzhong Lin, ${ }^{3}$ Bangwei Wu, ${ }^{2}$ Kunwu Yu, ${ }^{2}$ Zhaoqiang Dong, \\ Jianwei Zhang, ${ }^{4}$ Meng Chai, ${ }^{4}$ Yuyang Liu, ${ }^{4}$ Qingwei $J i,{ }^{2,3,4}$ and Yujie Zhou ${ }^{4}$
}

${ }^{1}$ Department of Cardiology, the Second Hospital of Shandong University, Jinan, China; ${ }^{2}$ Institute of Cardiovascular Diseases, Union Hospital, Tongji Medical College, Huazhong University of Science and Technology, Wuhan, China; ${ }^{3}$ Department of Cardiology, the People's Hospital of Guangxi Zhuang Autonomous Region, Nanning, China; and ${ }^{4}$ Department of Cardiology, Beijing Anzhen Hospital, Capital Medical University, Beijing Institute of Heart, Lung and Blood Vessel Disease, The Key Laboratory of Remodelingrelated Cardiovascular Disease, Ministry of Education, Beijing, China

\begin{abstract}
Valsartan has a protective effect against hypertension and atherosclerosis in humans and experimental animal models. This study aimed to determine the effect of prolonged treatment with angiotensin II (Ang II) on atherosclerosis and the effect of valsartan on the activity of CD4 ${ }^{+} \mathrm{T}$ lymphocyte subsets. The results showed that prolonged treatment (8 wks) with exogenous Ang II resulted in an increased atherosclerotic plaque size and a switch of stable-to-unstable plaque via modulating on CD4 ${ }^{+} \mathrm{T}$ lymphocyte activity, including an increase in the Thelper cell type 1 (Th1) and Th 17 cells and a decrease in Th2 and regulatory $T$ (Treg) cells. In contrast, valsartan treatment efficiently reversed the imbalance in CD4 ${ }^{+} \mathrm{T}$ lymphocyte activity, ameliorated atherosclerosis and elicited a stable plaque phenotype in addition to controlling blood pressure. In addition, treatment with anti-interleukin (IL)-5 monoclonal antibodies weakened the antiatherosclerotic effects of valsartan without affecting blood pressure.
\end{abstract}

Online address: http://www.molmed.org

doi: $10.2119 /$ molmed.2014.00195

\section{INTRODUCTION}

Cardiovascular diseases related to atherosclerosis continue to be the leading causes of illness and death among adults worldwide. Increased evidence has indicated that $\mathrm{CD}^{+} \mathrm{T}$ lymphocytes (also known as Th cells) are highly involved in atherosclerosis $(1,2)$. CD4 ${ }^{+} \mathrm{T}$ lymphocytes include effector T cells, which protect against pathogens, and regulatory $\mathrm{T}$ cells (Tregs), which protect against ef- fector responses to autoantigens and against responses to exogenous antigens that are harmful to the host. On the basis of their cytokine secretion profile, effector $\mathrm{T}$ cells are functionally subdivided into three types: T helper cell type 1 (Th1), Th2 and Th17. Over the past two decades, the Th1/Th2 paradigm has prevailed and the proatherogenic effect of Th1 cells was established (2-6). However, the role of Th2 cells in atherosclerosis re-

*KM, QZ, and QL contributed equally to this work.

Address correspondence to Yujie Zhou or Qingwei Ji, Department of Cardiology, Beijing Anzhen Hospital, Capital Medical University, Beijing Institute of Heart Lung and Blood Vessel Disease, The Key Laboratory of Remodeling-related Cardiovascular Disease, Ministry of Education, Beijing 100029, China. Phone: +86-10-64456488; Fax: 86-10-64456461;

E-mails: azzyj12@163.com (YJ), jqw124@163.com (QJ).

Submitted September 29, 2014; Accepted for publication February 9, 2015; Published Online (www.molmed.org) February 9, 2015.

\section{The Feinstein Institute for Medical Research \\ North ShoreLIJ}

mains uncertain (2). Recently, numerous studies have focused on Th17 in atherosclerosis, and most of these studies associated Th17 with the proatherogenic properties (7-9). The atheroprotective role of Tregs, including natural Tregs $\left(\mathrm{CD}^{+}{ }^{+} \mathrm{CD} 25^{+} \mathrm{FOXP3}^{+}\right.$Tregs ["FOXP3" being "forkhead box P3"]) and induced Tregs (iTregs), has been confirmed in numerous studies by using a variety of animal models (10-14).

Hypertension is one of the most important risk factors in the pathogenesis of atherosclerosis, and it has a synergistic effect with hyperlipidemia in promoting atherosclerosis. Angiotensin II (Ang II), the main effector of the renin-angiotensin system (RAS), is a bridge between hypertension and atherosclerosis, and it elicits a potent proatherogenic effect on the development and progression of atherosclerosis. Accumulating evidence has demonstrated that the effect of Ang II is mediated by Ang II type 1 (AT1) receptor activation, and AT1 receptor blockers 
(ARBs) are beneficial beyond their ability in lowering blood pressure; these benefits include antiinflammatory and antioxidative properties within the vasculature, resulting in the reduction of atherosclerotic progression and prevention of plaque rupture (15-17). Importantly, Ang II upregulates the Th1 and Th17 responses and suppresses the Th2 and Treg activity in the hypertensive model $(15,18-22)$. In contrast, reversing this modification significantly attenuates hypertension and target organ damage $(15,18-22)$.

Valsartan, a type of ARB, not only effectively regulates blood pressure but also reduces atherosclerotic events in patients with myocardial infarction and attenuates atherosclerosis in mice $(23,24)$. However, it remains uncertain whether the antiatherosclerotic effect of ARBs is associated with reversing the modification in $\mathrm{CD}^{+} \mathrm{T}$ lymphocyte subsets, including Th1, Th2 and Th17 cells and Tregs. Herein, we hypothesized that valsartan reduces the development of atherosclerosis by modulating the activity of $\mathrm{CD}^{+} \mathrm{T}$ lymphocyte subsets. In this study, using apolipoprotein E-deficient $\left(A p o E^{-/}\right)$ mice, we investigated the effect of prolonged treatment with Ang II (8 wks) on atherosclerosis and the effect of valsartan on the activity of $\mathrm{CD} 4^{+} \mathrm{T}$ lymphocyte subsets. In addition, the role of the Th2 immune response in valsartan-induced atheroprotection was investigated in the present study.

\section{MATERIALS AND METHODS}

\section{Animals and Treatment}

Male $A p o E^{-/}$mice with a C57BL/6 background were purchased from The Jackson Laboratory (Bar Harbor, ME, USA). These mice were bred and maintained in the Animal Center of Beijing University. These mice were maintained in a specific pathogen-free facility (Animal Center of Tongji Medical College of Huazhong University of Science and Technology, Wuhan, China). All protocols in this study were approved by the Animal Care and Use Committee of the
Union Hospital of Huazhong University of Science and Technology, China.

The 10-wk-old mice were fed a Westerntype diet containing $21 \%$ fat and $0.15 \%$ cholesterol. All mice underwent an implantation of osmotic minipumps (Alzet Model 2004, Alzet Corp., Palo Alto, CA, USA) for the infusion of Ang II (SigmaAldrich, St. Louis, MO, USA) at a rate of $1,000 \mathrm{ng} / \mathrm{kg} / \mathrm{min}$ or for saline as the control group. After 4 wks, when the minipumps were empty, we implanted new minipumps to continue the infusion of Ang II. The minipump implantation method has been described previously (19). Briefly, the mice were anesthetized with ketamine $(100 \mathrm{mg} / \mathrm{kg}$ ) and xylazine (10 mg/kg) via an intraperitoneal injection. After sterile preparation, osmotic minipumps were implanted via a $1-\mathrm{cm}$ incision between the scapulae. The incision was closed using surgical clips. Ang II was continuously infused for 8 wks. Mice were anaesthetized intraperitoneally using sodium pentobarbital at a concentration of $60 \mathrm{mg} / \mathrm{kg}$ and sacrificed.

\section{Animal Groups}

For the valsartan administration experiment, the animals were divided into the following three groups: group I $(n=10)$ served as the negative control group, which received a continuous administration of saline via minipumps; group II ( $n=10)$ received Ang II via minipumps and received a gavage of saline $(200 \mu \mathrm{L})$ once daily; and group III $(n=10)$ received Ang II via minipumps and received a gavage of valsartan $(10 \mathrm{mg} / \mathrm{kg}$, $200 \mu \mathrm{L}$ ) once daily.

To investigate whether the Th2 response mediated the atheroprotective effect of valsartan, the mice that were treated with Ang II plus valsartan were randomly grouped and injected intraperitoneally once per week for $8 w \mathrm{ks}$ with anti-interleukin (IL)-5 antibody (TRFK4; $50 \mu \mathrm{g}$ /injection) or control rat $\operatorname{IgG}_{2 \mathrm{~A}}$.

\section{Blood Pressure Measurement}

The blood pressure and heart rates of the mice were detected via the tail cuff method (BP-98A, Softron, Tokyo, Japan). Briefly, before measurement, the mice were placed in a dark cage at $37^{\circ} \mathrm{C}$ for $15 \mathrm{~min}$ on a heating pad. The tail-cuff pressure was continuously monitored, and signals from the pressure sensors were collected using a computer. All data were calculated from 10 readings for each animal.

\section{Antibodies and Reagents}

Ang II and valsartan were purchased from Sigma-Aldrich (St. Louis, MO, USA). Splenocytes were cultured in complete RPMI 1640 (Gibco [Thermo Fisher Scientific Inc., Waltham, MA, USA]) supplemented with $10 \%$ fetal calf serum (Gibco [Thermo Fisher Scientific]) and $100 \mathrm{U} / \mathrm{mL}$ streptomycin/penicillin. AntiCD4-fluorescein isothiocyanate (FITC) antibody, anti-CD25-allophycocyanin (APC) antibody, anti-interferon- $\gamma($ IFN- $\gamma)-$ phycoerythrin (PE) antibody, anti-IL-4-PE antibody, anti-IL-17A-PE antibody and anti-FOXP3-PE antibody were obtained from eBioscience (San Diego, CA, USA). Anti-monocyte/macrophage-2 (MOMA-2) antibody (lot number NG1904267) was purchased from Millipore (Billerica, MA, USA), and anti- $\alpha$-smooth muscle actin (SMA) was obtained from Abcam (Cambridge, MA, USA). Anti-matrix metalloproteinase (MMP)-2 antibody and antiMMP-9 antibody were obtained from Abcam, and anti-glyceraldehyde-3phosphate dehydrogenase (GAPDH) antibody was obtained from Antgene (Wuhan, China). Purified anti-CD4 antibody (clone RM4-5) was obtained from BD Systems (Bury St Edmunds, Suffolk, UK). Anti-IL-5 antibody and control rat IgG2A were obtained from R\&D Systems, (Minneapolis, MN, USA). All of the enzyme-linked immunosorbent assay (ELISA) cytokine detection kits were purchased from eBioscience.

\section{Weight and Lipids Measurement}

The weight of each mouse was measured weekly at the same time the blood pressure measurements were taken. Blood was collected from the angular vein every $4 \mathrm{wks}$, and the plasma was isolated by 
centrifugation at a rate of $1,200 \mathrm{~g}$ for 10 min at room temperature. The total cholesterol, triglyceride and high-density lipoprotein (HDL) cholesterol levels were measured by using enzymatic assays and determined with an autoanalyzer (Hitachi 917, Hitachi, Tokyo, Japan).

\section{Cytokine Assays}

The cytokine levels in the mouse plasma were measured by using ELISA according to the manufacturer's instructions (eBioscience). The cytokines included IFN- $\gamma$, IL-4, IL-5, IL-10, IL-13, IL-17, IL-18, IL-23, IL-33 and transforming growth factor (TGF)- $\beta 1$. The intraassay and interassay variation coefficients for all ELISA findings were $<10 \%$. All samples were measured in duplicate.

\section{Real-Time Polymerase Chain Reaction Analysis}

The aorta was extracted using TRIzol reagent (Invitrogen [Thermo Fisher Scientific]) according to the manufacturer's instructions. The total RNA was reversetranscribed using the RNA polymerase chain reaction (PCR) kit (Takara Biotechnology, Dalian, China). The mRNA was analyzed by real-time PCR by using the ABI PRISM 7900 Sequence Detector system (Applied Biosystems [Thermo Fisher Scientific]) according to the manufacturer's instructions. All reactions were performed in duplicate for each sample. The relative mRNA expression level was calculated by using the comparative computed tomography (CT) method formula $2^{-\triangle \triangle C T}$. Data were normalized to GAPDH. The primer pairs are listed in Table 1.

\section{Atherosclerotic Lesion Measurement \\ Atherosclerotic lesions were quantified in en face preparations of the whole aorta, and the frozen histological sections of the aortic sinus were processed as previously described (25). After en face aorta lesion staining, images of the entire vessel were captured by using a digital camera. After aortic sinus oil-red staining, all images were collected and analyzed by using the Image-Pro Plus 6.0 software (Media Cy- bernetics Inc., Rockville, MD, USA).}

Table 1. Real-time PCR primer sequences.

\begin{tabular}{|c|c|}
\hline Molecule & Sequence $\left(5^{\prime}-3^{\prime}\right)$ \\
\hline IFN- $\gamma$ forward & ACTGGCAAAAGGATGGTGAC \\
\hline IFN- $\gamma$ reverse & TGAGCTCATTGAATGCTTGG \\
\hline TBX21 forward & ATTGGTTGGAGAGGAAGCGG \\
\hline TBX21 reverse & TGTGCACCCTTCAAACCCTT \\
\hline IL-18 forward & ATGCTTTCTGGACTCCTGCC \\
\hline IL-18 reverse & GTCTGGTCTGGGGTTCACTG \\
\hline IL-4 forward & ACGAGGTCACAGGAGAAGGGA \\
\hline IL-4 reverse & AGCCCTACAGACGAGCTCACTC \\
\hline GATA3 forward & GCTACGGTGCAGAGGTATCC \\
\hline GATA3 reverse & TTCACACACTCССТGССТTC \\
\hline IL-5 forward & CACCGAGCTCTGTTGACAAG \\
\hline IL-5 reverse & TCCTCGCCACACTTCTCTTT \\
\hline IL-33 forward & GGTCCCGCCTTGCAAAATAA \\
\hline IL-33 reverse & CCGTGGATAGGCAGAGAAGT \\
\hline IL-17 forward & TCCAGAAGGCCCTCAGACTA \\
\hline IL-17 reverse & AGCATCTTCTCGACCCTGAA \\
\hline ROR $\gamma$ T forward & СTGTCCTGGGCTACССTACT \\
\hline ROR $\gamma$ T reverse & CCACTTGTTCCTGTTGCTGC \\
\hline IL-23 forward & AATAATGTGCCCCGTATCCA \\
\hline IL-23 reverse & CATGGGGCTATCAGGGAGTA \\
\hline TGF- $\beta 1$ forward & TGCTTCAGCTCCACAGAGAA \\
\hline TGF- $\beta 1$ reverse & TGGTTGTAGAGGGCAAGGAC \\
\hline IL-10 forward & ATAACTGCACCCACTTCCCA \\
\hline IL-10 reverse & GGGCATCACTTCTACCAGGT \\
\hline FOXP3 forward & САCСТATGCCACCCITATCC \\
\hline FOXP3 reverse & CGAACATGCGAGTAAACCAA \\
\hline MMP-2 forward & CCTTGTTACCATGGGTGGC \\
\hline MMP-2 reverse & AGCTCCTGGATCCCCTTGAT \\
\hline MMP-9 forward & AAAGGCAGCGTAGCCAGAA \\
\hline MMP-9 reverse & ACAACTCGTCGTCGTCGAAA \\
\hline GAPDH forward & AACTTTGGCATTGTGGAAGG \\
\hline GAPDH reverse & CACATTGGGGGTAGGAACAC \\
\hline
\end{tabular}

Primers were synthesized by an Invitrogen (Thermo Fisher Scientific) product.

For the immunohistochemical analysis of the lesion, approximately 5 - $\mu \mathrm{m}$ sections of aortic sinus were prepared. The antibodies used were as follows: purified anti-smooth muscle actin antibody (1:200) for smooth muscle cells, purified anti-MOMA-2 (1:200) for monocyte and macrophages, and purified anti-CD4 antibody (1:50) for T cells. Masson trichrome was used for the detection of collagen in the plaque area. Macrophages, smooth muscle cells and collagen were quantified by assessing the percent positive area of total plague for each marker, and $\mathrm{CD} 4^{+} \mathrm{T}$ cells were assessed by counting the number of cells stained positive per meter squared in plaque area.

\section{Western Blot}

Total protein of thoracic aorta was extracted by using a Total Protein Extraction Kit (Pierce [Thermo Fisher Scientific]). After centrifugation at $12,000 \mathrm{~g}$ for $30 \mathrm{~min}$ at $4^{\circ} \mathrm{C}$, the protein was obtained as the supernatant. The concentration of aorta protein was detected with the BCA Protein Assay Kit (Pierce [Thermo Fisher Scientific]). Samples containing $50 \mu \mathrm{g}$ protein were separated on a $10 \%$ sodium dodecyl sulfate-polyacrylamide gel electrophoresis and electrotransferred onto nitrocellulose membranes. Membranes were blocked by Tris-buffered saline with Tween containing 5\% skim milk. Then, the membranes were incubated with antiMMP-2, anti-MMP-9 and anti-GAPDH 
A

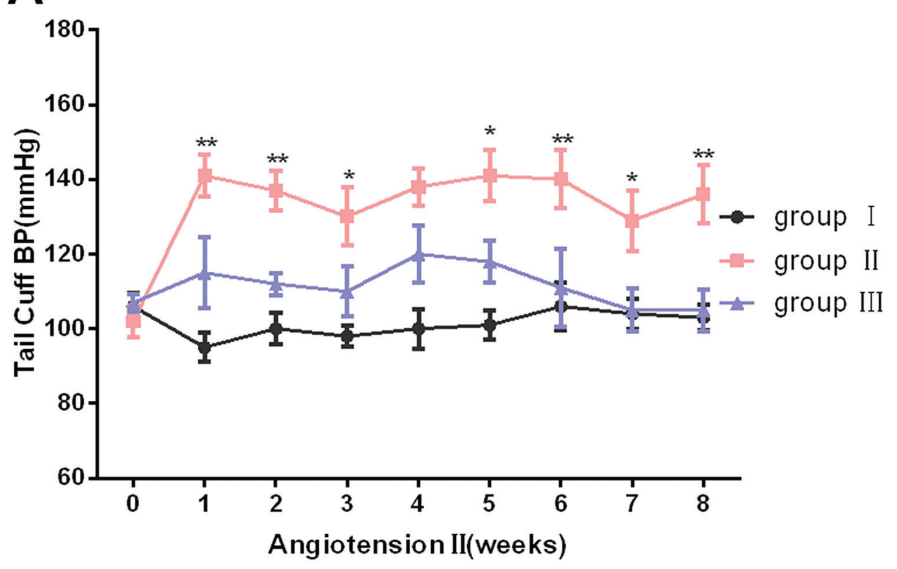

B

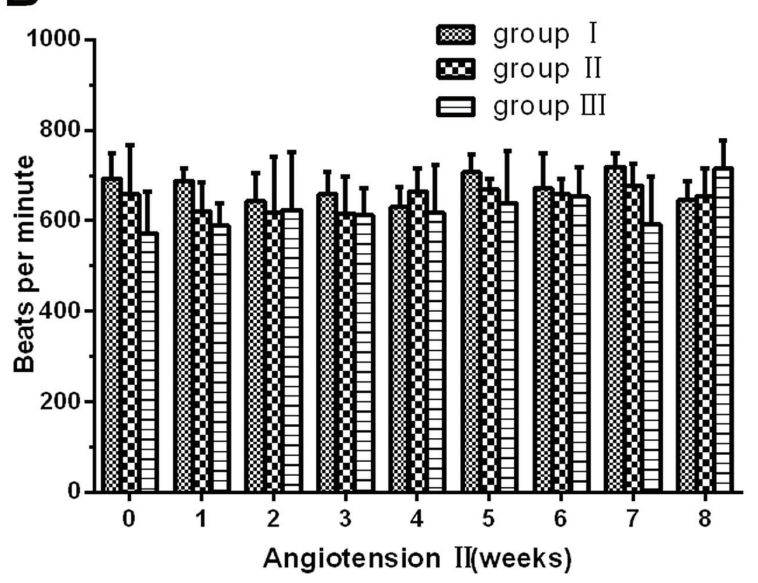

Figure 1. Blood pressure of Ang II infusion in $A p o E^{-/-}$mice. (A) Time course of systolic blood pressure (SBP) in the three groups. (B) Heart rate in the three groups. Data are expressed as the mean \pm standard error of the mean (SEM); $n=7-9 .{ }^{*} p<0.05$ versus group $\|$, ** $p<$ 0.01 versus group $\|$.

antibody at $4^{\circ} \mathrm{C}$ overnight. After being washed three times, the membranes were then incubated with secondary antibody for $2 \mathrm{~h}$. After washing, the membranes were finally developed with ECL reagent (Thermo Scientific [Thermo Fisher Scientific]). Data were analyzed using semiquantitative methods.

\section{Flow Cytometry}

To analyze Th1 $\left(\mathrm{CD} 4^{+} \mathrm{IFN}-\gamma^{+}\right)$, Th2 $\left(\mathrm{CD} 4^{+} \mathrm{IL}-4^{+}\right)$and Th17 $\left(\mathrm{CD} 4^{+} \mathrm{IL}-17^{+}\right)$, the splenic lymphocytes were isolated with Ficoll-Paque Plus (MP Biomedicals, Santa Ana, CA, USA)and suspended at a density of $2 \times 10^{6}$ cells $/ \mathrm{mL}$ in complete culture medium RPMI 1640. The cell suspen- sion in $1 \mathrm{~mL}$ medium was transferred to each well of 24-well plates. Cultures were stimulated with phorbol myristate acetate (PMA) (20 ng/mL), ionomycin $(1 \mu \mathrm{g} / \mathrm{mL})$ and monensin $(2 \mu \mathrm{mol} / \mathrm{mL})$. The incubator was set at $37^{\circ} \mathrm{C}$ under a $5 \% \mathrm{CO}_{2}$ environment. After $4 \mathrm{~h}$ of culture, the cells were collected for staining according to the manufacturer's instructions. Fixation and permeabilization were necessary before staining with IFN- $\gamma$, IL-4 or IL-17 antibody. To analyze $\mathrm{T}$ regulatory cells $\left(\mathrm{CD}^{+} \mathrm{CD}^{2} 5^{+} \mathrm{FOXP3}{ }^{+}\right)$, the cells were stained with anti-CD4 and anti-CD25 antibodies after fixation and permeabilization according to the manufacturer's instructions and were then stained with the
anti-FOXP3 antibody. Isotype controls were given to correct compensation and confirm antibody specificity. Data were collected by using a FACSCalibur flow cytometer (BD Biosciences, San Jose, CA, USA) and analyzed with FlowJo software (Treestar, Inc., Ashland, OR, USA).

\section{Statistical Analysis}

The data are shown as the mean \pm standard deviation (SD) unless otherwise indicated. Comparisons between groups were performed using the Student $t$ test when the data were normally distributed and the group variances were equal. The Mann-Whitney rank sum test was used when the data were not normally distributed or if the group variances were unequal. One-way analysis of variance was used for multiple comparisons among three groups, followed by the Bonferroni test when the data were normally distributed and the group variances were equal. The Kruskal-Wallis test followed by the Dunn test was used when the group data were not normally distributed or if the group variances were unequal. All statistical analyses were performed by using the GraphPad Prism 6.0 software (GraphPad Software Inc., La Jolla, CA, USA). $p<$ 0.05 was considered significant.

\section{RESULTS}

\section{Effect of Valsartan on Blood Pressure}

We detected the blood pressure of the three groups of $A p o E^{-/}$mice each week using the tail-cuff method. At baseline, the systolic blood pressure did not demonstrate a difference in the three groups. The systolic blood pressure increased markedly with Ang II infusion after $1 \mathrm{wk}$. The blood pressure of group II (Ang II + saline) remained high throughout the experimental course. The blood pressure level of group III, which was given valsartan, was significantly lower than that of group II (Figure 1A). The difference in the systolic pressure between groups I and III was not statistically significant. In addition, there was no significant difference in the heart rate in the three groups (Figure 1B). 


\section{Effect of Valsartan on Body Weight and Plasma Lipids}

The results of body weight and plasma lipids analysis, including the total plasma cholesterol, HDL cholesterol and triglycerides, were similar in the three groups (Table 2). These results indicated that the administration of valsartan had no obvious effect on the body weight or plasma lipid levels.

\section{Valsartan Ameliorates Atherosclerosis in Ang II-Treated $\mathrm{ApoE}^{-/-}$Mice}

The results showed that the atherosclerosis lesion size in the aortic sinus of group II was significantly increased compared with that of group I $(698,000 \pm$ 210,700 versus $405,000 \pm 175,000 \mu \mathrm{m}^{2}, p<$ 0.05 ; Figure 2C). In contrast, there was a $35 \%$ reduction in the atherosclerosis lesion size in mice given valsartan (group III) $\left(458,500 \pm 84,350 \mu^{2}\right)$ compared with group II ( $p<0.05$; Figure $2 \mathrm{C})$. We also quantified the lesion area in en face preparations of the entire aorta. We found an increased plaque burden in group II compared with group I $(41.84 \pm 13.17 \%$ versus $18.76 \pm 4.58 \%, p<0.01$; Figure 2D). However, there was a significant reduction in the aortic plaque burden in group III compared with group II $(26.51 \pm 5.22 \%$ versus $41.84 \pm 13.17 \%, p<0.05$; Figure 2D).

\section{Effects of Valsartan on the Formation of Atherosclerotic Plaques}

To investigate the effects of the administration of valsartan on Ang II-treated ApoE $E^{-/}$mice, we performed immunohistochemical studies, including staining for $\alpha$-SMA, CD4 and macrophages as well as the use of Masson trichrome staining of the atherosclerosis lesions in the aortic sinus of the three groups. The $\alpha$-SMA-positive area in group II was decreased compared with group I (18.36 \pm $4.68 \%$ versus $30.78 \pm 6.88 \%, p<0.01$; Figure $3 \mathrm{~B})$, particularly at the surface of the lesion cap (Figure 3A). However, the valsartan-administered group (group III) increased in the positive area of $\alpha$-SMA compared with group II $(26.80 \pm 6.30 \%$ versus $18.36 \pm 4.68 \%, p<0.05$; Figure $3 \mathrm{~B})$; the positive area on the lesion cap also
Table 2. Body weight and lipid levels in groups I-III.

\begin{tabular}{lccccc}
\hline & Age & Group I & Group II & Group III & $P$ \\
\hline Body weight (g) & & & & & \\
& 10 wks & $21.55 \pm 1.88$ & $21.04 \pm 2.22$ & $20.69 \pm 1.99$ & 0.94 \\
& 18 wks & $29.44 \pm 1.72$ & $28.57 \pm 1.56$ & $29.18 \pm 2.73$ & 0.69 \\
Triglycerides (mmol/L) & 10 wks & $0.88 \pm 0.11$ & $0.68 \pm 0.21$ & $0.79 \pm 0.15$ & 0.35 \\
& 18 wks & $1.53 \pm 0.16$ & $1.67 \pm 0.17$ & $1.61 \pm 0.21$ & 0.43 \\
Total cholesterol (mmol/L) & & & & & \\
& 10 wks & $17.32 \pm 3.23$ & $16.98 \pm 2.51$ & $17.65 \pm 4.86$ & 0.39 \\
HDL cholesterol (mmol/L) & 18 wks & $21.20 \pm 4.17$ & $20.55 \pm 3.41$ & $20.41 \pm 5.11$ & 0.94 \\
& 10 wks & $0.53 \pm 0.12$ & $0.58 \pm 0.09$ & $0.63 \pm 0.07$ & 0.52 \\
& 18 wks & $0.28 \pm 0.07$ & $0.28 \pm 0.06$ & $0.31 \pm 0.12$ & 0.79 \\
\hline
\end{tabular}

Values are expressed as means $\pm \mathrm{SD} ; \mathrm{n}=6$.

\section{A}

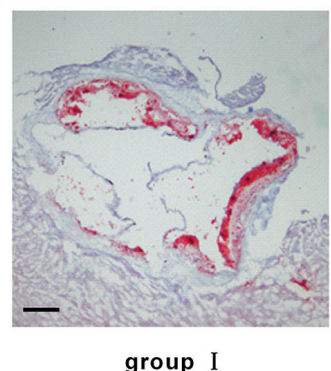

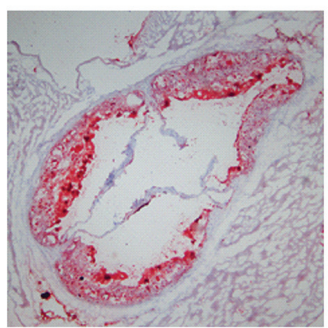

group II

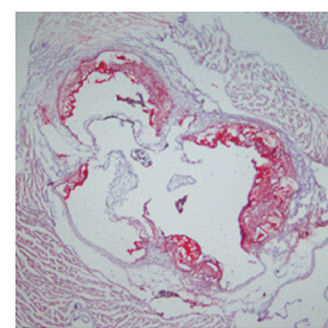

group III

C
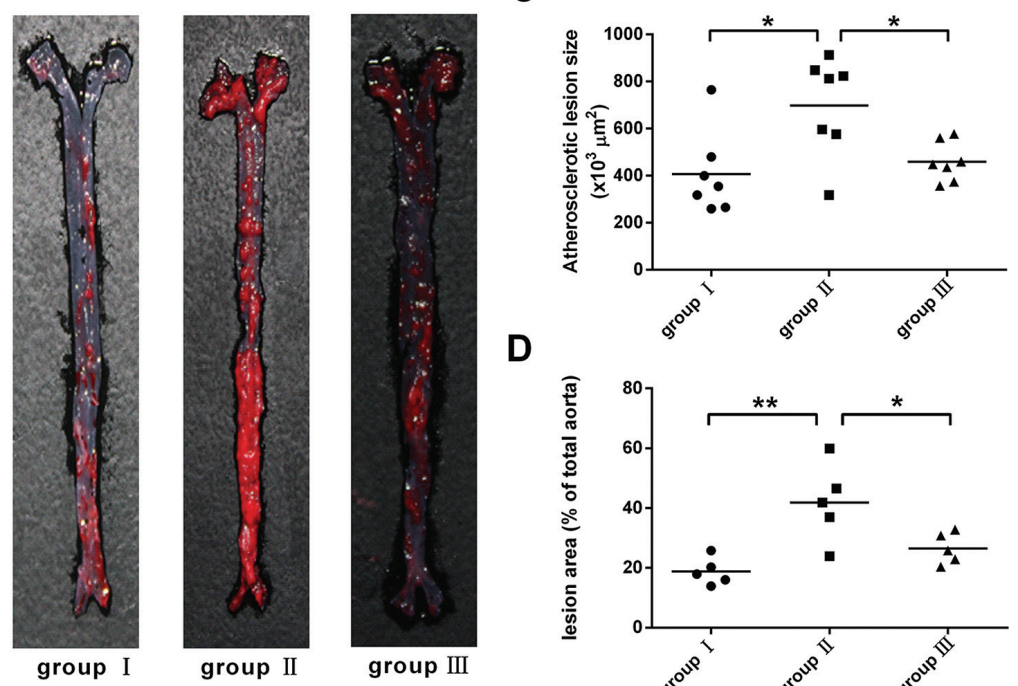

D

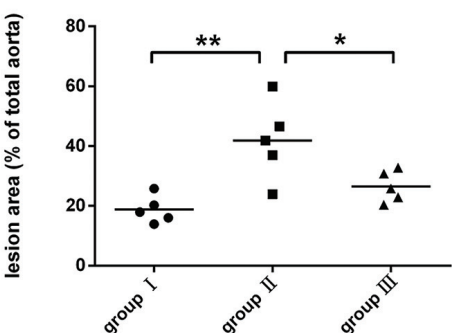

Figure 2. Valsartan ameliorates the development of atherosclerosis in Ang II-treated $A p o E^{-/-}$mice (I). (A) Representative sections of aortic sinus stained with oil red $O$ in the three groups. Black bar $=200 \mu \mathrm{m}$. (B) Representative photographs of oil red $\mathrm{O}$ staining in the surface lesion area of the entire aorta. (C) Quantitative analysis of the lesion size of aortic sinus staining in the three groups $\left({ }^{*} P<0.05\right.$ versus group II). (D) Quantitative analysis of the percent lesion area of the entire vessel area $\left({ }^{*} p<0.05\right.$ versus group $\|{ }^{* *} p<0.01$ versus group II). The values are presented as the mean $\pm S D ; n=5-7$. 

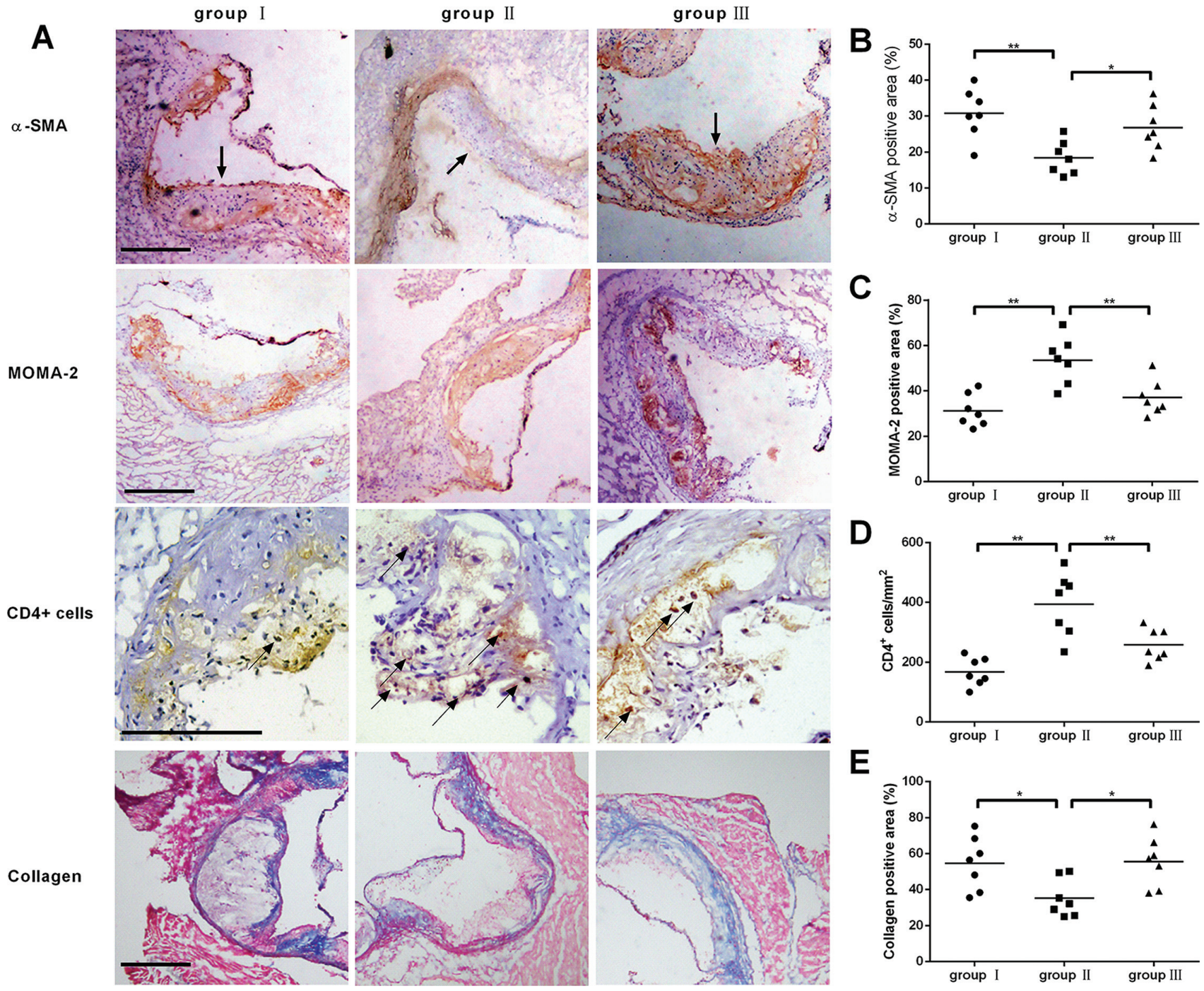

Figure 3. Valsartan ameliorates the development of atherosclerosis in Ang Il-treated $A p o E^{-/-}$mice (II). (A) Representative sections of aortic sinus stained with antibodies to $\alpha$-SMA, MOMA-2, CD4 and collagen staining in ApoE $E^{-/-}$mice in the three groups. Black bar $=200 \mu \mathrm{m}$. (B-E) Quantitative analysis of $\alpha$-SMA, MOMA-2, CD4 and collagen staining in the three groups $\left({ }^{*} p<0.05\right.$ versus group II, ** $p<0.01$ versus group II). The values are presented as the mean $\pm S D ; n=7$.

increased (Figure 3A). The macrophages and CD4 staining in group II were increased compared with those in group I $(53.55 \pm 10.31 \%$ versus $31.21 \pm 1.12 \%$ for macrophages, $p<0.01 ; 383.50 \pm 112.00$ versus $171.00 \pm 50.69 / \mathrm{mm}^{2}$ for $\mathrm{CD}^{+}$ T cells, $p<0.01$; Figures $3 C$, D). The administration of valsartan reversed the modification between group III and group II (37.08 $\pm 7.69 \%$ versus $53.55 \pm$ $10.31 \%$ for macrophage, $p<0.01 ; 262.30$ \pm 57.07 versus $383.50 \pm 112.00 / \mathrm{mm}^{2}$ for $\mathrm{CD}^{+} \mathrm{T}$ cells, $p<0.05$; Figures $\left.3 \mathrm{C}, \mathrm{D}\right)$.
The Masson staining for collagen in group II was decreased compared with group I $(35.19 \pm 10.56 \%$ versus $54.58 \pm$ $14.84 \%, p<0.05)$, and the administration of valsartan reversed the difference between groups III and II (55.54 $\pm 13.79 \%$ versus $35.19 \pm 10.56 \%, p<0.05$, Figure $3 \mathrm{E}$ ) .

We used real-time PCR and Western blot analysis to measure the expression of $M M P-2$ and MMP-9 of the aorta in the three groups. Figure 4 showed that the mRNA and protein expression of $M M P-2$ and $M M P-9$ in group II increased com- pared with group I, and the administration of valsartan reversed the modification. Taken together, the results indicated that Ang II induces plaque instability, whereas valsartan promotes a stable plaque phenotype.

\section{Valsartan Modulates the Activity of $\mathrm{CD4}^{+} \mathrm{T}$ Lymphocyte Subsets}

Mazzolai demonstrated that endogenous Ang II elicits a Th1 response in $A p o E^{-/-}$mice (5). The results of the present study showed that there was not 
only an imbalance of Th1/Th2 but that the imbalance of Th17/Treg was also worsened in the exogenous Ang II-treated ApoE ${ }^{-/}$mice. As shown in Figure 5 , the frequencies of Th1 cells (CD4 ${ }^{+}$IFN- $\gamma^{+}$T cells) and Th17 cells $\left(\mathrm{CD} 4^{+} \mathrm{IL}-17^{+} \mathrm{T}\right.$ cells) were increased in group II compared with group I (27.13 \pm $4.96 \%$ versus $17.32 \pm 3.88 \%$ for Th1, $p<$ $0.01 ; 2.13 \pm 0.42 \%$ versus $0.95 \pm 0.31 \%$ for Th17, $p<0.01)$. In contrast, the frequencies of Th2 (CD4 ${ }^{+} \mathrm{IL}^{+}{ }^{+} \mathrm{T}$ cells) and Treg $\left(\mathrm{CD}^{+} \mathrm{CD} 25^{+} \mathrm{FOXP3}^{+} \mathrm{T}\right.$ cells) cells were decreased in group II compared with group I ( $1.60 \pm 0.27 \%$ versus $2.30 \pm 0.35 \%$ for Th2, $p<0.05 ; 7.13 \pm 1.18 \%$ versus $10.53 \pm 1.79 \%$ for Treg, $p<0.05)$. However, valsartan reversed these results and showed lower frequencies of Th1 and Th17 cells with higher frequencies of Th2 cells and Tregs compared with group II $(18.73 \pm 3.17 \%$ versus $27.13 \pm 4.96 \%$ for Th1, $p<0.01 ; 2.73 \pm 0.61 \%$ versus $1.60 \pm$ $0.27 \%$ for Th $2, p<0.01 ; 1.07 \pm 0.42 \%$ versus $2.13 \pm 0.42 \%$ for Th17, $p<0.01$; and $10.25 \pm 1.80 \%$ versus $7.13 \pm 1.18 \%$ for Tregs, $p<0.05)$.

\section{Valsartan Attenuates Both Systemic and Local Inflammation}

To examine the effect of the administration of valsartan on systemic inflammation, we analyzed the cytokine levels in the serum of the three groups. Group II had a higher level of IFN- $\gamma$, IL-18, IL-17 and IL-23 with a lower level of IL-4, IL-5, IL-33, TGF- $\beta 1$ and IL-10 compared with group I (Figures 6A, B). However, animals in group III had the opposite results compared with group II (Figures 6A, B). In addition, we analyzed the mRNA expression of these cytokines in the aorta of the mice. Our results showed that group II had a higher level of IFN- $\gamma$, T box-21 (TBX21), IL-18, retinoid acid receptor-related orphan receptor gamma $\mathrm{t}(R O R \gamma T), I L-17$ and $I L-23$ and a lower level of $I L-4$, GATA binding protein 3 (GATA3), IL-5, IL-10, IL-33, TGF- $\beta 1$ and FOXP3 compared with group I (Figures 6C, D). Interestingly, mice treated with valsartan (group III) showed opposite results compared with
A

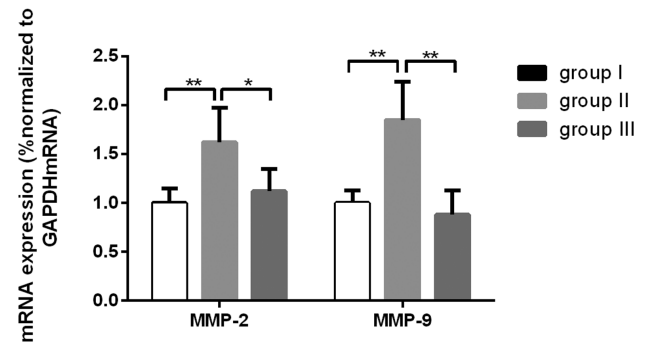

B

C
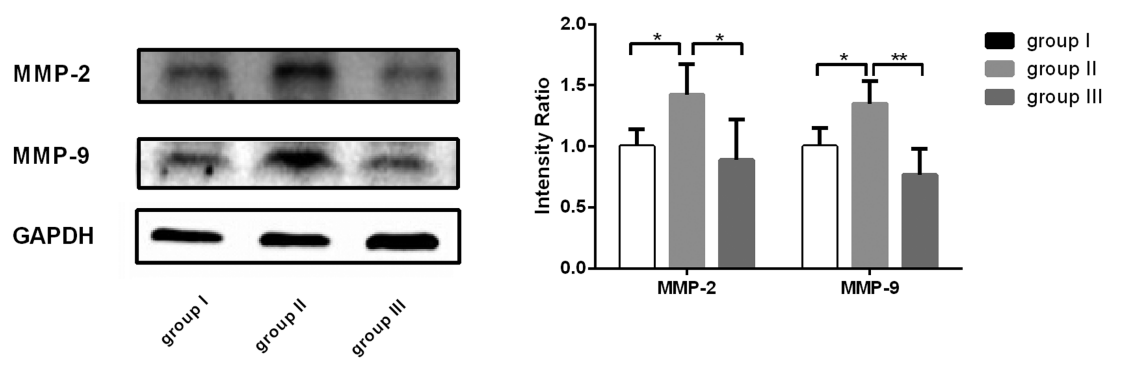

Figure 4. Valsartan reduces the expression of MMP-2 and MMP-9. (A) mRNA expression of MMP-2 and MMP-9 in the three groups. (B, C) Protein expression of MMP-2 and MMP-9. Treatment with Ang II induced mRNA and protein expression of MMP-2 and MMP-9, which were significantly increased compared with the control group in the aorta of the ApoE ${ }^{-/-}$ mice. Group I was set as the control group $\left({ }^{*} p<0.05\right.$ versus group II, ${ }^{* *} p<0.01$ versus group II). The values are presented as the mean $\pm S D ; n=4-5$.

group II (Figures 6C, D). However, the levels of IL-13, another Th2-related cytokine, were below the detection limit in the three groups.

\section{Anti-IL-5 Monoclonal Antibodies Abrogate the Effect of Valsartan on Atherosclerosis}

We reported that valsartan upregulated the frequencies of Th2 cells. Furthermore, the ELISA and PCR results for cytokines also indicated that Th2 cell-related cytokines, particularly IL-5, were significantly increased after valsartan treatment. Several studies reported the atheroprotective role of IL-5 in both $\mathrm{LDLR}^{-/-}$mice and IL-33-treated ApoE ${ }^{-/-}$ mice $(26,27)$. To determine whether the Th2 response exhibits a vital role of preventing plaque formation in valsartantreated $A p o E^{-/-}$mice, we performed an IL-5 neutralization study. The mice were treated in the same way as mice in group III (Ang II plus valsartan), and they were subsequently injected with anti-IL-5 monoclonal antibody (mAb) or control rat IgG2A once per week. We measured the IL- 5 level in the serum between the groups, and the level of IL-5 was normalized in the anti-IL- 5 group compared with the control group (Figure 7A). Although there was no difference in blood pressure between the groups (Figure 7B), we found that anti-IL-5 mAb reversed the protective effect of valsartan on plague growth (Figure 8). Moreover, although the $\alpha$-SMA and Masson staining of plaques were not significantly different between the groups, macrophage infiltration was increased in the anti-IL-5 $\mathrm{mAb}$ group compared with the control group.

\section{DISCUSSION}

In the present study, we found that 8 wks of exogenous Ang II treatment not only accelerated the development of atherosclerosis but that it also promoted a switch from a stable-to-unstable plaque that is associated with a change in $\mathrm{CD} 4^{+}$ 
A
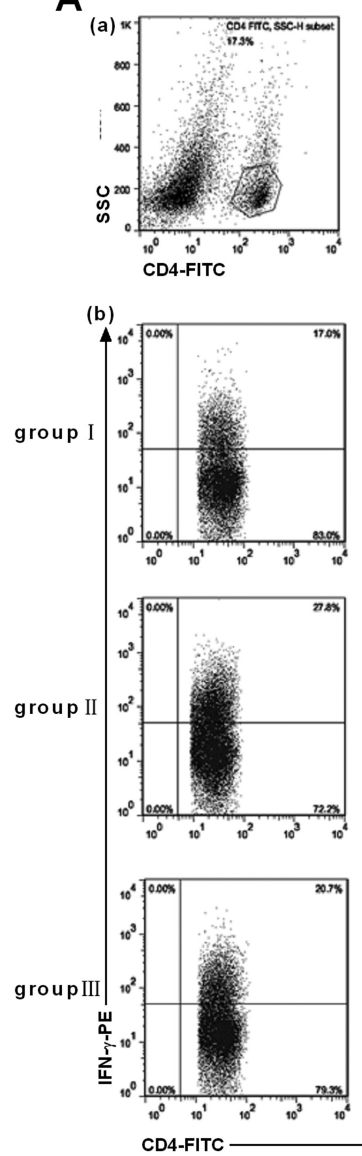

(c)
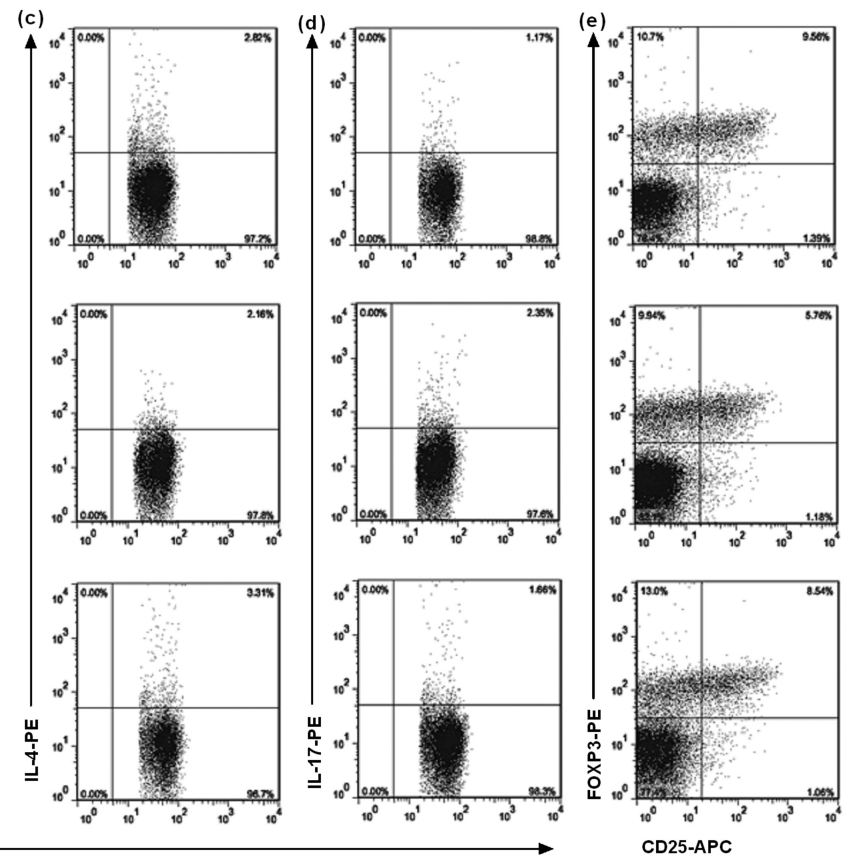

B

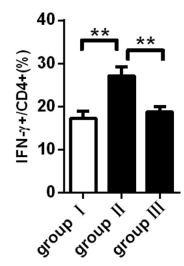

C

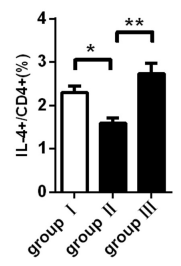

D

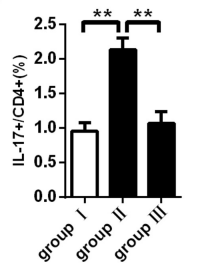

E

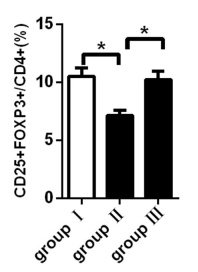

Figure 5. Effect of valsartan administration on Th1, Th2 and Th17 cells and Tregs in the spleen of Ang II-treated ApoE ${ }^{-/-}$mice. (A) CD4 ${ }^{+}$T-cell subsets were gated (a). Representative pictures of Th1 cells (b), Th2 cells (c), Th17 cells (d) and Tregs (e) are shown. The numbers in the upper right quadrants indicate positive percentages of these cells. (B) Results of statistical analysis of Th1 cells ( ${ }^{*} p<0.01$ versus group II). (C) Results of statistical analysis of Th2 cells ( ${ }^{*} p<0.05$ versus group II; ${ }^{* *} p<0.01$ versus group II). (D) Results of statistical analysis of Th17 cells ( ${ }^{* *} p<0.01$ versus group II). (E) Results of statistical analysis of Tregs $\left({ }^{*} P<0.05\right.$ versus group II). The values are presented as the mean $\pm S D ; n=5-7$.

T lymphocyte activity. We also found that valsartan reversed the effect of Ang II on the progress of atherosclerosis and modulated the activity of $\mathrm{CD} 4^{+} \mathrm{T}$ lymphocytes. The administration of valsartan significantly increased the frequencies of Th2 and Treg cells, which was accompanied by a high expression of GATA3 and FOXP3 mRNA and a high level of their characteristic cytokines, including IL-4, IL-5, IL-10, IL-33 and TGF- $\beta 1$. In contrast, valsartan treatment significantly de- creased the frequencies of Th1 and Th17 cells, which was accompanied by a low expression of T-bet and ROR $\gamma \mathrm{T}$ mRNA and a low level of their characteristic cytokines, including IFN- $\gamma$, IL-18, IL-17 and IL-23. Treatment with anti-IL-5 mAb weakened the antiatherosclerotic effects of valsartan without affecting blood pressure, suggesting that valsartan attenuated atherosclerosis, at least in part, by upregulating the Th2 immune response. These results also indicated a novel antiatherosclerotic mechanism of valsartan beyond blood pressure control.

Numerous studies have demonstrated the proatherogenic properties of Th1 by using different atherosclerosis-prone models (2-6). Evidence from clinical investigations has shown that the upregulated Th1 immune response is overwhelming in patients with acute coronary syndrome $(28,29)$ and that the imbalance between Th1 and Th2 might play a role in plaque rupture $(3,5,30)$. Interestingly, changes in the Th2 but not Th1 levels have been correlated with cardiovascular events (31). Engelbertsen et al. (31) measured the baseline circulating Th1 and Th2 levels and the production of cytokines in 700 participants who were enrolled in the Malmö Diet and Cancer Study. The results indicated that high Th2 levels were independently associated with a reduced risk of acute myocardial infarction in women and that the secretion of IL-4 was negatively correlated with the onset of cardiovascular disease, suggesting a protective role of the Th2 response in atherosclerosis-related disease. These results were consistent with Tracy's report (32), in which circulating Th1 levels were positively associated with the intimal media thickness of the common carotid, whereas circulating Th2 levels were negatively associated with the intimal media thickness of the common carotid.

Because of the atherogenic role of IL-4 in $A p o E^{-/-} I L-4^{-/-}$mice (33), Th2 cells are thought to play a pathogenic role in atherosclerosis. In addition, some studies have shown that the Th2 response does not change either in atherosclerosis- 
A

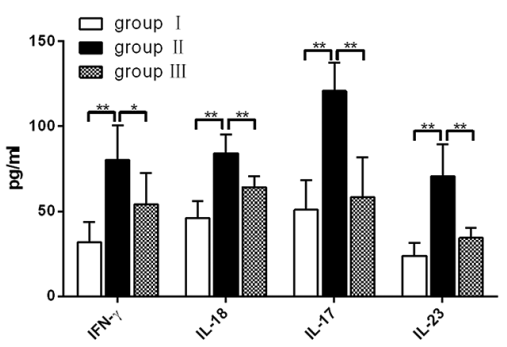

C

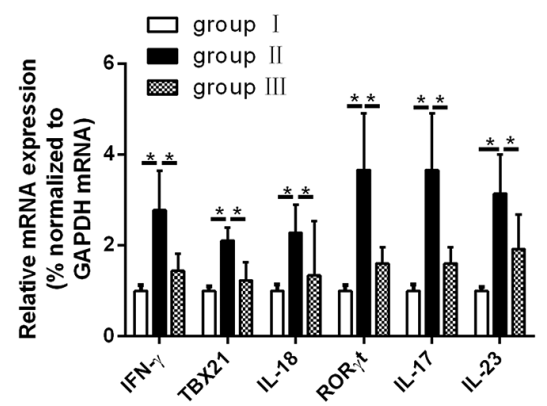

Figure 6. The levels of serum cytokines among the three groups and the mRNA expression in the aorta. (A) The levels of serum Th1-and Th17-related cytokines, including IFN- $\gamma$, IL-18, IL-17 and IL-23, in the three groups. (B) The levels of serum Th2- and Treg-related cytokines, including IL-4, IL-5, IL-33, IL-10 and TGF- $\beta 1$, in the three groups. (C) Expression of IFN- $\gamma$, TBX21, IL-18, ROR 7 , IL-17 and IL-23 mRNA in aorta in the three groups. (D) Expression of IL-4, GATA3, IL-5, IL-33, IL-10, TGF- $\beta 1$ and FOXP3 mRNA in aorta in the three groups. The values are presented as the mean $\pm \mathrm{SD} ; \mathrm{n}=5-7$. ${ }^{*} p<0.05$ versus group $\|$, ${ }^{* *} p<0.01$ versus group $\|, " p>0.05$ versus group II.

prone models or in patients with coronary artery disease, suggesting that Th2 may function as a bystander in atherosclerosis $(5,6,28,29)$. However, an increasing number of studies have confirmed the antiatherogenic properties of the Th2 response $(26,27,34-36)$.

In the present study, $8 \mathrm{wks}$ of exogenous Ang II treatment significantly upregulated the Th1 response and suppressed the Th2 response; therefore, the imbalance of Th1 and Th2 was significantly enhanced in Ang II-treated $A p o E^{-/}$mice. In contrast, blockade of the AT1 receptor by using valsartan efficiently attenuated Ang II-induced atherosclerosis and modulated the imbalance of Th1 and Th2, which was characterized by decreased Th1 levels and increased Th2 levels. In light of this observation, the production of Th2-related cytokines, including IL-4, IL-5, IL-13 and IL33, was measured. The results showed that the IL-4 levels were increased ap-
B

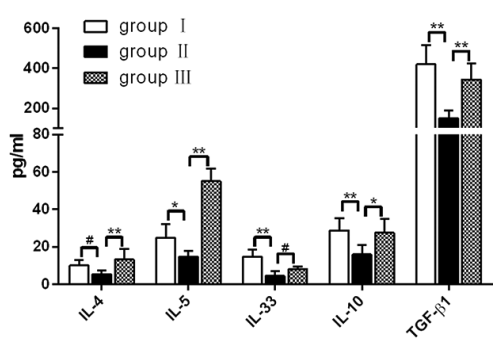

D

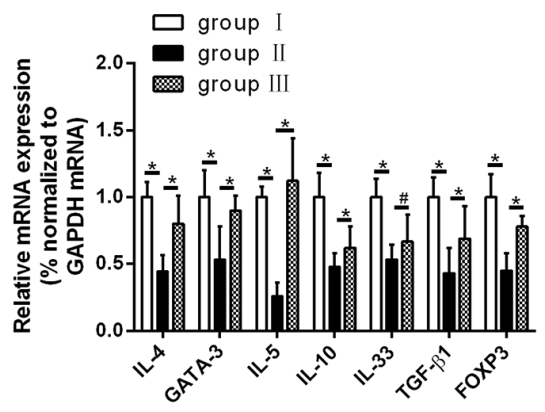

proximately twofold, and the IL-5 levels were increased approximately fivefold; the IL-33 levels were also significantly increased in valsartan-treated mice, al-
A

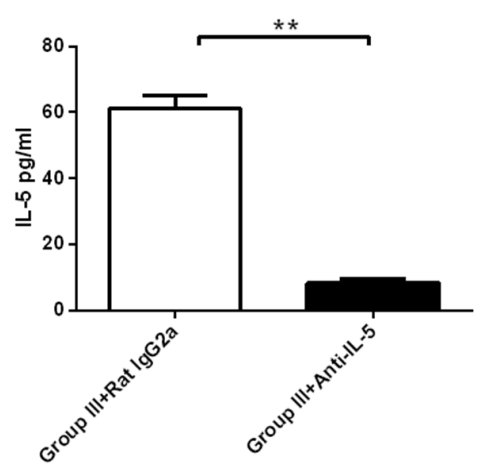

though the levels of IL-13 were below the detection limit among the three groups. With respect to the maximum rate of increase in the IL-5 level, and because IL-5 played a critical role in both $L D L R^{-/}$mice and IL-33-treated $A p o E^{-/-}$mice $(26,27,34,35)$, the anti-IL-5 mAb was administered to block the Th2-mediated effect in the present study. Although blood pressure was not affected, treatment with anti-IL-5 mAb significantly promoted the development of atherosclerosis, suggesting that Th2 plays a protective role in Ang II-induced atherosclerosis and participates in the antiatherosclerotic process induced by valsartan.

Consistent with previous reports (19), the Th17 immune response was found to be upregulated by Ang II in the present study. Madhur et al. (19) first demonstrated that Ang II induced a Th17 response and that IL-17 deficiency in mice results in a significant decrease in blood pressure and vascular inflammation, which strongly indicates the pathogenic role of the Th17 response in Ang II-induced hypertension. Because the $\mathrm{CD} 4^{+} \mathrm{T}$ lymphocyte response plays a critical role in Ang II-induced hypertension and atherosclerosis, it was hypothesized that the Th17 response also plays a pathogenic role in Ang II-induced atherosclerosis $(5,21,36-40)$. However, Madhur et al. (41) found that IL-17 deficiency did not af-

\section{B}

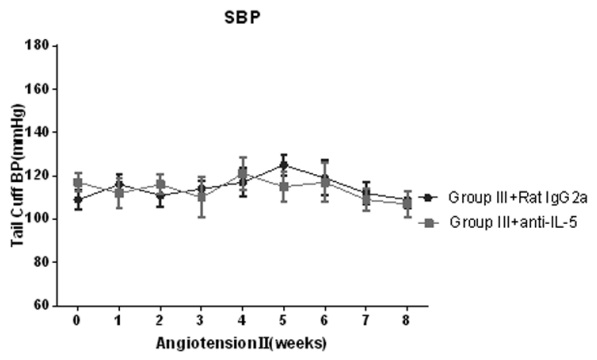

Figure 7. (A) The levels of serum IL-5 in the two groups ${ }^{* *} p<0.01$ versus Ang $I^{+} \mathrm{Val}{ }^{+}$Rat lgG2a). (B) Blood pressure of two groups of $\mathrm{ApoE}^{-/-}$mice. Time course of systolic blood pressure in the three groups is shown. Data are expressed as the mean \pm SEM; $n=5-6$. SBP, systolic blood pressure. 


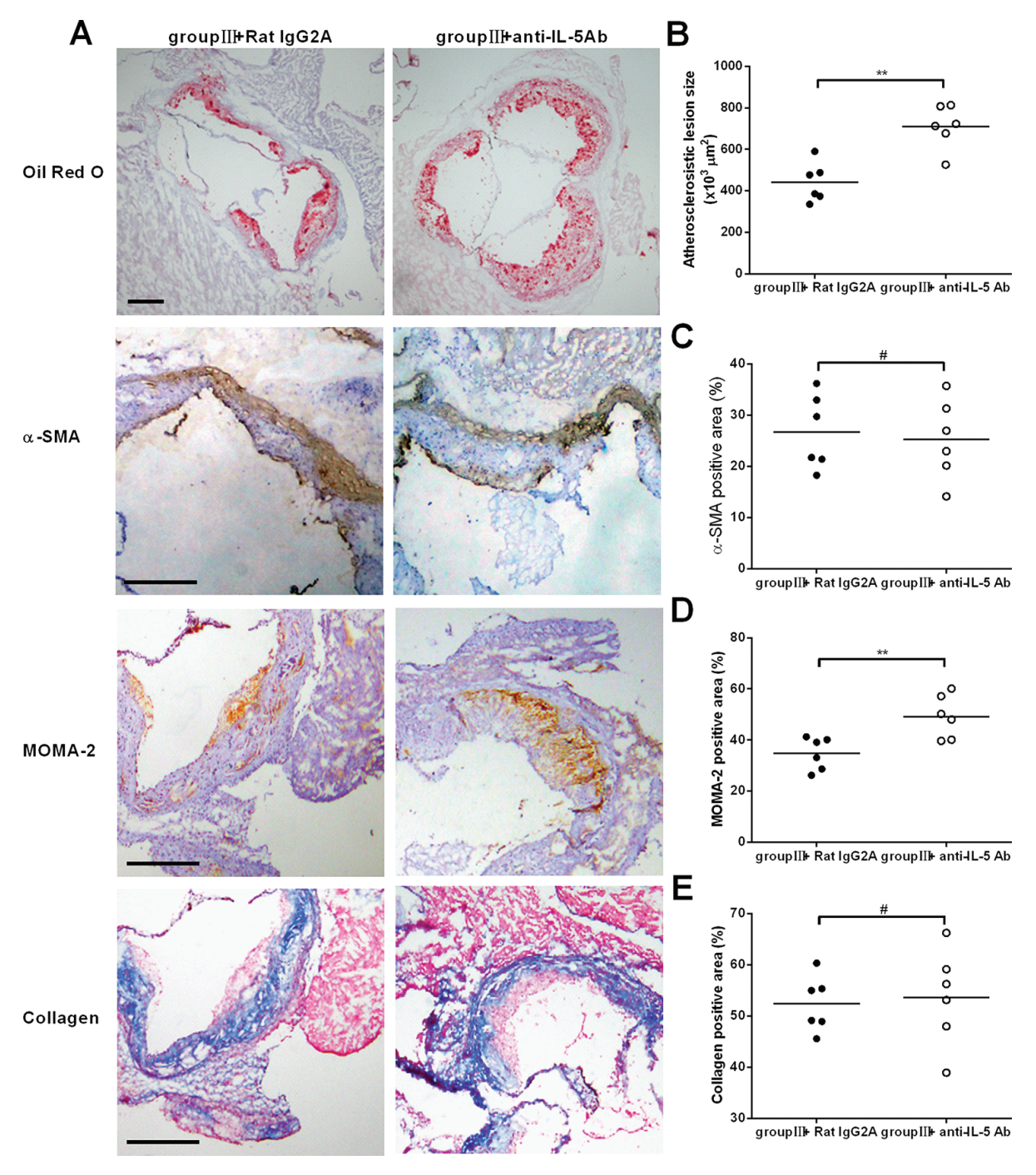

Figure 8. Anti-IL-5 mAb abrogates the effect of valsartan on atherosclerosis in Ang II-treated $A p o E^{-/-}$mice. (A) Representative sections of the aortic sinus stained with oil red $O$, and antibodies specific to $\alpha$-SMA, MOMA-2 and collagen in ApoE $E^{-/-}$mice. Black bar $=200 \mu \mathrm{m}$. (B-E) Quantitative analysis of the lesion size, $\alpha$-SMA and MOMA-2 staining. The values are presented as the mean $\pm S D ; n=6 .{ }^{* *} p<0.01,{ }^{*} p>0.05$.

fect the lesion size induced by 4 wks of Ang II infusion. This study revealed that IL-17 might be associated with plaque stability because IL-17 deficiency resulted in a decrease in leukocyte and dendritic cell infiltration and in aortic superoxide production.

In the present study, we found that upregulation of the Th17 immune response was accompanied by an increase in lesion size and plaque instability in 8-wk Ang II-treated ApoE ${ }^{-/-}$mice. When valsartan was used to block the effect of
Ang II, the results also showed the reduction in Th17 and IL-17 levels, lesion size and plaque stability. Because other cells such as $\gamma \delta \mathrm{T}$ cell also secrete IL-17 and Th17 secrete other cytokines other than IL-17, there are many confounders in previous studies, and those results do not adequately represent the role of the Th17 immune response in atherosclerosis. Therefore, the question of whether the Th17 immune response plays a role in lesion size and plaque stability in prolonged Ang II-treated $A p o E^{-/-}$mice re- quires further investigation. The best way to answer this question is adoptive transfer of Th17 to the Ang II-induced atherosclerosis prone model.

Changes in the Treg cell levels induced by Ang II in $A p o E^{-/}$mice were measured for the first time in the present study. The Treg levels, the expression of FOXP3 and the production of IL- 10 and TGF- $\beta 1$ were significantly blunted in Ang II-treated mice, whereas valsartan treatment efficiently restored Treg activity. Because abundant studies have shown the protective role of Treg cells in both atherosclerosis and Ang II-induced target-organ damage (40,42-44), the Treg response may play a protective role in Ang II-induced atherosclerosis. However, there is still insufficient direct evidence supporting that Treg cells protect mice from Ang II-induced atherosclerosis, and this point needs to be elucidated in future research.

\section{CONCLUSION}

In summary, prolonged treatment with Ang II resulted in an increased atherosclerotic plaque size and a switch from stable-to-unstable plaques via modulation of $\mathrm{CD}^{+} \mathrm{T}$ lymphocyte activity. However, whether Ang II binds to the AT1 receptor in the $\mathrm{T}$ cell directly by regulating T-cell polarization is still unclear and should be investigated in the future. In contrast, valsartan treatment efficiently reversed the imbalance in $\mathrm{CD}^{+} \mathrm{T}$ lymphocyte activity, ameliorated atherosclerosis and elicited a stable plaque phenotype, and these effects were partially mediated by the Th2 immune response.

\section{ACKNOWLEDGMENTS}

This work was supported by the National Natural Science Foundation of China (81160045, 81270285, 81270354 and 81360055), the Beijing Municipal HighLevel Talent Foundation of Health System (2011-1-5), Beijing Municipal Administration of Hospitals Clinical Medicine Development of Special Funding Support (Code ZY201303) and the National Key Clinical Specialty Construction Project. 


\section{DISCLOSURE}

The authors declare that they have no competing interests as defined by Molecular Medicine, or other interests that might be perceived to influence the results and discussion reported in this paper.

\section{REFERENCES}

1. Medzhitov R, et al. (2011) Highlights of 10 years of immunology in Nature Reviews Immunology. Nat. Rev. Immunol. 11:693-702.

2. Robertson AK, Hansson GK. (2006) T cells in atherogenesis: for better or for worse? Arterioscler. Thromb. Vasc. Biol. 26:2421-32.

3. Frostegård J, et al. (1999) Cytokine expression in advanced human atherosclerotic plaques: dominance of pro-inflammatory (Th1) and macrophage-stimulating cytokines. Atherosclerosis. 145:33-43.

4. Whitman SC, Ravisankar P, Elam H, Daugherty A. (2000) Exogenous interferon-gamma enhances atherosclerosis in apolipoprotein E-/- mice. Am. J. Pathol. 157:1819-24.

5. Mazzolai L, et al. (2004) Endogenous angiotensin II induces atherosclerotic plaque vulnerability and elicits a Th1 response in ApoE-/- mice. Hypertension. 44:277-82.

6. Cheng $X$, et al. (2008) Suppressive oligodeoxynucleotides inhibit atherosclerosis in ApoE(-/-) mice through modulation of Th1/Th2 balance. J. Mol. Cell Cardiol. 45:168-75.

7. Erbel C, et al. (2009) Inhibition of IL-17A attenuates atherosclerotic lesion development in apoEdeficient mice. J. Immunol. 183:8167-75.

8. Smith E, et al. (2010) Blockade of interleukin-17A results in reduced atherosclerosis in apolipoprotein E-deficient mice. Circulation. 121:1746-55.

9. Butcher MJ, Gjurich BN, Phillips T, Galkina EV. (2012) The IL-17A/IL-17RA axis plays a proatherogenic role via the regulation of aortic myeloid cell recruitment. Circ. Res. 110:675-87.

10. Ait-Oufella H, et al. (2006) Natural regulatory

$\mathrm{T}$ cells control the development of atherosclerosis in mice. Nat. Med. 12:178-80.

11. Mor A, et al. (2007) Role of naturally occurring CD4+ CD25+ regulatory T cells in experimental atherosclerosis. Arterioscler. Thromb. Vasc. Biol. 27:893-900.

12. Zhong $\mathrm{Y}$, et al. (2012) CD4+LAP+ and CD4+CD25+Foxp3+ regulatory $\mathrm{T}$ cells induced by nasal oxidized low-density lipoprotein suppress effector $\mathrm{T}$ cells response and attenuate atherosclerosis in ApoE-/- mice. J. Clin. Immunol. 32:1104-17

13. Yu K, et al. (2013) Thymic stromal lymphopoietin attenuates the development of atherosclerosis in ApoE-/- mice. J. Am. Heart Assoc. 2:e000391.

14. Klingenberg R, et al. (2013) Depletion of FOXP3+ regulatory $\mathrm{T}$ cells promotes hypercholesterolemia and atherosclerosis. J. Clin. Invest. 123:1323-34.
15. Strawn WB, Ferrario CM. (2002) Mechanisms linking angiotensin II and atherogenesis. Curr. Opin. Lipidol. 13:505-12.

16. Ferrario CM. (2002) Use of angiotensin II receptor blockers in animal models of atherosclerosis. Am. J. Hypertens. 15:9S-13S.

17. Arishiro K, et al. (2007) Angiotensin receptor-1 blocker inhibits atherosclerotic changes and endothelial disruption of the aortic valve in hypercholesterolemic rabbits. J. Am. Coll. Cardiol. 49:1482-9.

18. Tedgui A, Mallat Z. (2006) Cytokines in atherosclerosis: pathogenic and regulatory pathways. Physiol. Rev. 86:515-81.

19. Madhur MS, et al. (2010) Interleukin 17 promotes angiotensin II-induced hypertension and vascular dysfunction. Hypertension. 55:500-7.

20. Barhoumi T, et al. (2011) T regulatory lymphocytes prevent angiotensin II-induced hypertension and vascular injury. Hypertension. 57:469-76.

21. Kassan M, Galan M, Partyka M, Trebak M, Matrougui K. (2011) Interleukin-10 released by $\mathrm{CD} 4^{+} \mathrm{CD} 25^{+}$natural regulatory $\mathrm{T}$ cells improves microvascular endothelial function through inhibition of NADPH oxidase activity in hypertensive mice. Arterioscler. Thromb. Vasc. Biol. 31:2534-42.

22. Shao J, et al. (2003) Imbalance of T-cell subsets in angiotensin II-infused hypertensive rats with kidney injury. Hypertension. 42:31-8.

23. McMurray J, et al. (2006) The effect of valsartan, captopril, or both on atherosclerotic events after acute myocardial infarction: an analysis of the Valsartan in Acute Myocardial Infarction Trial (VALIANT). J. Am. Coll. Cardiol. 47:726-33.

24. de Gasparo M. (2000) New basic science initiatives with the angiotensin II receptor blocker valsartan. J. Renin Angiotensin Aldosterone Syst. 1:S3-5.

25. Daugherty A, Whitman SC. (2003) Quantification of atherosclerosis in mice. Methods Mol. Biol. 209:293-309.

26. Binder CJ, et al. (2004) IL-5 links adaptive and natural immunity specific for epitopes of oxidized LDL and protects from atherosclerosis. J. Clin. Invest. 114:427-37.

27. Miller AM, et al. (2008) IL-33 reduces the development of atherosclerosis. J. Exp. Med. 205:339-46.

28. Methe H, et al. (2005) Enhanced T-helper-1 lymphocyte activation patterns in acute coronary syndromes. J. Am. Coll. Cardiol. 45:1939-45.

29. Ji QW, et al. (2009) Downregulation of T helper cell type 3 in patients with acute coronary syndrome. Arch. Med. Res. 40:285-93.

30. Benagiano M, et al. (2003) T helper type 1 lymphocytes drive inflammation in human atherosclerotic lesions. Proc. Natl. Acad. Sci. U. S. A. 100:6658-63.

31. Engelbertsen D, et al. (2013) T-helper 2 immunity is associated with reduced risk of myocardial infarction and stroke. Arterioscler. Thromb. Vasc. Biol. 33:637-44.

32. Tracy RP, et al. (2013) T-helper type 1 bias in healthy people is associated with cytomegalovirus serology and atherosclerosis: the Multi-Ethnic Study of Atherosclerosis. J. Am. Heart Assoc. 2:e000117.
33. Davenport P, Tipping PG. (2003) The role of interleukin-4 and interleukin-12 in the progression of atherosclerosis in apolipoprotein E-deficient mice. Am. J. Pathol. 163:1117-25.

34. Perry HM, et al. (2013) Helix-loop-helix factor inhibitor of differentiation 3 regulates interleukin-5 expression and B-1a B cell proliferation. Arterioscler. Thromb. Vasc. Biol. 33:2771-9.

35. Cardilo-Reis L, et al. (2012) Interleukin-13 protects from atherosclerosis and modulates plaque composition by skewing the macrophage phenotype. EMBO Mol. Med. 4:1072-86.

36. McLaren JE, et al. (2010) IL-33 reduces macrophage foam cell formation. J. Immunol.185:1222-9.

37. Guzik TJ, et al. (2007) Role of the T cell in the genesis of angiotensin II induced hypertension and vascular dysfunction. J. Exp. Med. 204:2449-60.

38. Marvar PJ, et al. (2012) T lymphocytes and vascular inflammation contribute to stress-dependent hypertension. Biol. Psychiatry. 71:774-82.

39. Zhang JD, et al. (2012) A novel role for type 1 angiotensin receptors on $\mathrm{T}$ lymphocytes to limit target organ damage in hypertension. Circ. Res. 110:1604-17.

40. Barhoumi T, et al. (2011) T regulatory lymphocytes prevent angiotensin II-induced hypertension and vascular injury. Hypertension. 57:469-76.

41. Madhur MS, et al. (2011) Role of interleukin 17 in inflammation, atherosclerosis, and vascular function in apolipoprotein e-deficient mice. Arterioscler. Thromb. Vasc. Biol. 31:1565-72.

42. Kvakan H, et al. (2009) Regulatory T cells ameliorate angiotensin II-induced cardiac damage. Circulation. 119:2904-12.

43. Matrougui K, et al. (2011) Natural regulatory T cells control coronary arteriolar endothelial dysfunction in hypertensive mice. Am. J. Pathol. 178:434-41.

44. Ait-Oufella H, et al. (2013) Natural regulatory T cells limit angiotensin II-induced aneurysm formation and rupture in mice. Arterioscler. Thromb. Vasc. Biol. 33:2374-9.

Cite this article as: Meng K, et al. (2015) Valsartan attenuates atherosclerosis via upregulating the Th2 immune response in prolonged angiotensin II-treated ApoE ${ }^{-/-}$mice. Mol. Med. 21:143-53. 